UNIVERSIDADE DE SÃO PAULO
FACULDADE DE CIÊNCIAS FARMACÊUTICAS DE RIBEIRÃO PRETO

MAÍRA ROCHA DE SOUZA PRADO

Avaliação dos efeitos deletérios de fármacos psicotrópicos sobre o desenvolvimento dos estágios embrio-larvais de zebrafish 


\title{
Avaliação dos efeitos deletérios de fármacos psicotrópicos sobre o desenvolvimento dos estágios embrio-larvais de zebrafish
}

\author{
Dissertação de Mestrado \\ apresentada ao Programa de Pós- \\ Graduação em Toxicologia da \\ Faculdade de Ciências \\ Farmacêuticas de Ribeirão \\ Preto/USP para obtenção do Título de \\ Mestre em Ciências \\ Área de Concentração: Toxicologia. \\ Orientado: Maíra Rocha de Souza Prado \\ Orientadora: Profa. Dra. Danielle Palma de Oliveira
}

Versão corrigida da Dissertação de Mestrado apresentada ao Programa de PósGraduação em Toxicologia da Faculdade de Ciências Farmacêuticas de Ribeirão Preto/USP em 02/03/2018. Aversão original encontra-se disponível na Faculdade de Ciências Farmacêuticas de Ribeirão Preto/USP. 
AUTORIZO A REPRODUÇÃO E DIVULGAÇÃO TOTAL OU PARCIAL DESTE TRABALHO, POR QUALQUER MEIO CONVENCIONAL OU ELETRÔNICO, PARA FINS DE ESTUDO E PESQUISA, DESDE QUE CITADA A FONTE.

Avaliação dos efeitos deletérios de fármacos psicotrópicos sobre 0 desenvolvimento dos estágios embrio-larvais de zebrafish 
AUTORIZO A REPRODUÇAÕ E DIVULGAÇÃO TOTAL OU PARCIAL DESTE TRABALHO, POR QUALQUER MEIO CONVENCIONAL OU ELETRÔNICO, PARA FINS DE ESTUDO E PESQUISA, DESDE QUE CITADA A FONTE.

Prado, Maíra Rocha de Souza

Avaliação dos efeitos deletérios de fármacos psicotrópicos sobre o desenvolvimento dos estágios embrio-larvais de zebrafish. Ribeirão Preto, 2018.

86p. : il. ; $30 \mathrm{~cm}$.

Dissertação de Mestrado, apresentada à Faculdade de Ciências Farmacêuticas de Ribeirão Preto/USP - Área de concentração: Biociências Aplicadas à Farmácia (Toxicologia).

Orientador: Oliveira, Danielle Palma de.

1. Fármacos psicotrópicos. 2. Danio rerio. 3. Teratogênese. 4. Neurotoxicidade 


\section{RESUMO}

PRADO, M. R. de S. Avaliação dos efeitos deletérios de fármacos psicotrópicos sobre o desenvolvimento dos estágios embrio-larvais de zebrafish. 2018. 86f. Dissertação (Mestrado). Faculdade de Ciências Farmacêuticas de Ribeirão Preto - Universidade de São Paulo, Ribeirão Preto, 2018.

Nos últimos anos, a prescrição e o consumo de medicamentos psicotrópicos vêm aumentando ao redor do mundo, crescendo assim a presença destes compostos em efluentes. Embora os esgotos domésticos e hospitalares em geral sejam encaminhados para as Estações de Tratamento de Esgotos (ETEs), o tratamento convencional aplicado não é eficiente na remoção da maioria dos fármacos e, consequentemente, estes poluentes alcançam os corpos hídricos. Frente a essas informações, é importante entender os possíveis danos causados tanto para seres humanos, como para o ecossistema como um todo, após a exposição a esses fármacos, por meio da água contaminada. Desta maneira, nossa hipótese é que os fármacos psicotrópicos presentes em amostras de água possam induzir a danos neuronais e no desenvolvimento de organismos não alvo, como peixes. Para responder a esta hipótese, avaliamos os efeitos teratogêncios e neurotóxicos de fármacos psicotrópicos como venlafaxina, haloperidol, carbamazepina e fluoxetina, por meio da análise de parâmetros de letalidade e sub-letalidade nos em Danio rerio, visando a determinação dos possíveis efeitos teratogênicos. Adicionalmente, utilizamos a determinação da atividade da enzima acetilcolinesterase como biomarcador de neurotoxicidade em larvas, após a exposição dos embriões e a avaliação do padrão de movimentação. Os fármacos foram testados individualmente e em misturas, para avaliar os efeitos da co-exposição, simulando um cenário mais real de contaminação aquática. Os resultados mostraram que os fármacos carbamazepina e venlafaxina não apresentaram nenhum dos efeitos analizados de forma estatisticamente significativa. Já os fármacos haloperidol e fluoxetina, mesmo não apresentando efeito sobre a teratogenicidade e movimentação, provocaram um aumento na atividade enzimática, em doses consideradas bastante baixas. Considerando que esta estimulação inicial voltou a níveis próximos ao controle negativo e, no caso da fluoxetina, um estudo inibição da atividade em doses mais altas, sugerimos a manifestação de hormesis. A mistura dos fármacos não apresentou efeito significativo nas avaliações realizadas, mostrando que o aumento da atividade enzimática dfoi antagonizado na quando em mistura. Nosso trabalho mostra a importância do estudo de toxicidade em doses baixas, para que uma correta avaliação do risco seja possível.

Palavras-chave: Venlafaxina, haloperidol, carbamazepina; fluoxetina; Danio rerio; teratogênese; neurotoxicidade. 


\begin{abstract}
PRADO, M. R. de S. Evaluation of the deleterious effects of psychotropic drugs on the development of the embryonic stages of zebrafish. 2018. 86f. Dissertation (Master). School of Pharmaceutical Sciences of Ribeirão Preto University of São Paulo, Ribeirão Preto, 2018.
\end{abstract}

In the last years, the prescription and use of psychotropic drugs have being increasing around the world, consequently the discharge of these drugs and their metabolites have also increased in wastewater. Considering the presence in the environment and the toxic effects to target and non target species, these compounds were classified as emerging water contaminants. The main sources of these compounds are the domestic and hospital treated effluents, because the conventional treatment applied by effluent treatment plants is not efficient to remove most of drugs, and consequently these pollutants reach the water bodies. In this context, it is important to understand the possible damages to humans and ecosystems after exposure to these drugs through contaminated water. In that way, the hypothesis of this research is that psychotropic drugs present in water samples could induce neurologic and development damages in non-target organisms, such as fish. Therefore, we propose the evaluation of teratogenic and neurotoxic effects of the psychotropic drugs Fluoxetine, Carbamazepine, Venlafaxine and Haloperidol using the determination of the activity of cholinesterase enzyme as biomarker of neurotoxicity in larvae, after the exposure of Danio rerio eggs. Lethality and sub-lethality parameters will be also analyzed in the same organisms to establish the potential of teratogenic effects, the movement analysis was also performed. These drugs will be tested individually and in mixtures to evaluate the effects of coexposure simulating a real scenario of water pollution. Carbamazepine and venlafaxine don't responde with significant results in any of these evaluations. Haloperidol and Fluoxetine presented negative results in teratogenic and moviment effects, but they incresed the activity of acetylcholinesterase enzime, this result was different from another study, that these drugs in highest concentrations decrease this activity, so it can indicate the presence of hormesis effect. However, the mixtures of drugs don't present any significant effect on all evaluations, this result can suggest that these effects caused by haloperidol and fluoxetine on the enzime activity were canceled, it can be a interaction of antagonist effects on the mixtures.

Keywords: Venlafaxine; haloperidol; carbamazepine; fluoxetine; Danio rerio; teratogenesis; neurotoxicity. 


\section{INTRODUÇÃO}

\subsection{Fármacos psicotrópicos}

Nos últimos anos, tem-se observado um crescente uso de medicamentos, dentre eles, pode-se ressaltar os fármacos psicotrópicos, usados para controle e tratamento de diversas doenças psiquiátricas. De acordo com o Instituto Americano IMS Health e a Comissão Internacional de Direitos Humanos dos Cidadãos em Saúde Mental (CCHR, do inglês: Citizens Commission on Human Rights), em 2013 aproximadamente 25\% da população dos Estados Unidos fazia uso de algum medicamento psiquiátrico (CCHR, 2013).

Dentro do painel nacional, o uso desta classe de medicamento também é bastante frequente. Um levantamento realizado com pacientes atendidos nas unidades de atenção primária básica de saúde de algumas capitais do Brasil entre 2009 e 2010 revelou que mais de $50 \%$ dos pacientes das cidades do Rio de Janeiro, São Paulo, Fortaleza e Porto Alegre apresentavam algum tipo de transtorno psiquiátrico (GONÇALVES et. al., 2014). Ainda no cenário brasileiro, em 2015, um estudo feito durante um mês na maior cidade do país, a prevalência de fármacos psicotrópicos em São Paulo foi de 5,89\% dos participantes, sendo parte deles sem diagnóstico de doença psiquiátrica (QUINTANA et al., 2015). Esse vasto aumento pode ser atribuído a alguns fatores, como a maior frequência nos diagnósticos, a introdução de novos medicamentos na área farmacêutica e as novas indicações terapêuticas para fármacos psicotrópicos já existentes, bem como o estresse da vida moderna (RODRIGUES MAP, 2006; ALFENA, M. D., 2015).

Dentre as doenças psiquiátricas, os quadros de depressão e ansiedade são os mais comuns, podendo atingir cerca de 10 a 15\% da população em algum momento da vida (O'DONNELL, J. M.; SHELTON, R. C., 2012). Segundo a Organização Mundial da Saúde (OMS, do inglês: World Health Organization, WHO), atualmente 300 milhões de pessoas ao redor do mundo são acometidas pela depressão, tendo sido registrado um aumento de $18 \%$ na incidência deste transtorno entre 2005 e 2015 (UN, 2017). Ainda de acordo com a OMS, em 2008, O Brasil contribui com $20,3 \%$ da estimativa global de doenças neuropsiquiátricas (WHO, 2011). 
Transtornos mentais estão entre as doenças que mais incapacitam a população, de acordo com a Dataprev (Empresa de Tecnologia e Informações da Previdência Social), 4\% do total de auxílios-doença concedidos no ano de 2016 foram por afastamento dos trabalhadores devido a transtornos mentais e de comportamento, sendo $80 \%$ dos casos devido à depressão (BRASIL, 2017).

Dentro deste contexto, um indicador da alta prevalência de doenças psiquiátricas é o aumento vertiginoso no consumo de medicamentos. Segundo o anuário estatístico do mercado farmacêutico de 2015 da ANVISA (Agência Nacional de Vigilância Sanitária), os medicamentos com o maior faturamento atuam sobre o sistema nervoso central (SNC), contribuindo com $14,3 \%$ na receita do setor (BRASIL, 2016).

Frente a essas estatísticas alarmantes sobre transtornos psiquiátricos e uso de medicamentos, a OMS lidera há um ano uma campanha sobre saúde mental. As 2 milhões de visitas ao website desta campanha mostram o grande interesse mundial sobre esse assunto (WHO, 2017).

A depressão é caracterizada por alguns sintomas como humor deprimido, diminuição do interesse por atividades comuns e falta de concentração, já os episódios de ansiedade abrangem o transtorno de ansiedade generalizada, transtorno obsessivo-compulsivo e quadros de pânico, todos esses sintomas causados por alterações na neurotransmissão noradrenérgica e serotonérgica (O'DONNELL, J. M.; SHELTON, R. C., 2012). Além da depressão e ansiedade, outros transtornos mentais também comuns são os de mania e psicose, caracterizados por senso inexistente ou distorcido da realidade, podendo ser causados por uma hiperatividade de dopamina (O'DONNELL, J. M.; SHELTON, R. C., 2012).

\subsubsection{Fármacos psicotrópicos estudados}

Para 0 tratamento das doenças mentais relacionadas aos neurotransmissores, existe uma gama de medicamentos disponibilizados pela indústria farmacêutica. Os medicamentos psicotrópicos mais comuns são os inibidores seletivos de recaptação de serotonina (do inglês, selective serotonin re-uptake inhibitors - SSRIs), como a fluoxetina, comumente utilizada para 
tratamento de depressão, desordens de pânico e perturbação obsessivacompulsiva (CALISTO; ESTEVES, 2009).

Como alternativa aos SSRIs, os fármacos inibidores seletivos de recaptação tanto de serotonina como norepinefrina, a venlafaxina por exemplo, são prescritos para quadros de depressão e ansiedade em casos em que os SSRIs não apresentam eficiência adequada (CALISTO; ESTEVES, 2009).

Ainda entre os psicotrópicos, destacamos a carbamazepina, um anticonvulsivante amplamente utilizado em crises convulsivas tônico-clônicas, proporcionando resposta no SNC, porém sem levar à depressão respiratória (MIAO, X.; METCALFE, C. D. 2003; DE ALMEIDA, 2013). Ademais, o antipsicótico típico haloperidol é usado em tratamentos de pacientes com quadros de esquizofrenia e distúrbios sanguíneos cerebrais. Trata-se de um fármaco antagonista dopaminérgico, e por ter esse mecanismo de ação, tem como evento adverso os efeitos extrapiramidais (SAWADA et al., 2000; GIACOMINI et al., 2006; BRUNTON; CHABNER; KMOLLMANN, 2012).

$\mathrm{Na}$ Tabela 1 são mostradas algumas características fisico-químicas e farmacocinéticas desses fármacos, objetos de estudo do presente trabalho.

Tabela 1 - Características fisico-químicas e farmacocinéticas dos fármacos venlafaxina, haloperidol, carbamazepina e fluoxetina (THUMEL et. al., 2012).

\begin{tabular}{ccccccc}
\hline & $\begin{array}{c}\text { Massa } \\
\text { molar } \\
(\mathbf{g} / \mathbf{m o l})\end{array}$ & LogP & Metabolismo & $\begin{array}{c}\text { Tempo } \\
\text { de meia- } \\
\text { vida } \\
\text { (horas) }\end{array}$ & $\begin{array}{c}\text { Ligação } \\
\text { plasmática } \\
(\%)\end{array}$ & $\begin{array}{c}\text { Excreção } \\
\text { urinária } \\
(\%)\end{array}$ \\
\hline $\begin{array}{c}\text { Venlafaxina } \\
\mathrm{C}_{17} \mathrm{H}_{27} \mathrm{NO}_{2}\end{array}$ & 277.408 & 2.9 & CYP2D6 & 4,9 & 27 & 4,6 \\
$\begin{array}{c}\text { Haloperidol } \\
\mathrm{C}_{21} \mathrm{H}_{23} \mathrm{ClFNO} \mathrm{F}_{2}\end{array}$ & 375,9 & 3.2 & $\begin{array}{c}\text { CYP3A4 } \\
\text { CYP2D6 }\end{array}$ & 18 & 92 & $<2,5$ \\
$\begin{array}{c}\text { Carbamazepina } \\
\mathrm{C}_{15} \mathrm{H}_{12} \mathrm{~N}_{2} \mathrm{O}\end{array}$ & 236.274 & 2.5 & CYP3A4 & 53 & 94 & 1 \\
$\begin{array}{c}\mathrm{Fluoxetina}_{\mathrm{C}} \\
\mathrm{C}_{17} \mathrm{H}_{18} \mathrm{~F}_{3} \mathrm{NO}\end{array}$ & 309.332 & 4 & CYP2D6 & 15 & 74 & $<1$ \\
\hline
\end{tabular}

Estes quatro fármacos estão na lista de substâncias entorpecentes, psicotrópicos, precursoras e outras sob controle especial, da Portaria número 
344/98 da Secretaria de Vigilância Sanitária/Ministério da Saúde, sendo todos classificados como substâncias sujeitas a receita médica com controle especial (RDC 49) (BRASIL, 2015). Ainda assim, ocorre o uso indiscriminado e não consciente desses medicamentos. Os ansiolíticos e antipsicóticos são usados de forma mais cautelosa, pelo potencial de dependência, porém alguns antidepressivos, como a fluoxetina, são muitas vezes considerados como a "droga da felicidade", sendo disseminado para a população como inofensivo (MENDONÇA et al., 2009). Uso inadequado de fluoxetina muitas vezes é comum para auxiliar no processo de perda de peso. Em um estudo feito entre $2005 \mathrm{e}$ 2006 na cidade de Santo André, SP, foram analisadas receitas especiais em farmácias magistrais e drogarias, mostrando que $27 \%$ das prescrições continham esse medicamento, sendo essas, em sua maioria, destinadas a mulheres (quase $80 \%$ ). O estudo mostrou também que houve um grande número de receitas com associação de fluoxetina a substâncias anorexígenas, como anfetaminas (CARLINI et al., 2009; ANVISA, 2010). 


\subsection{Fármacos psicotrópicos como contaminantes ambientais emergentes}

Existem mais de 129 milhões de compostos orgânicos e inorgânicos conhecidos e registrados no Chemical Abstract Service (CAS 2017), sendo que a maioria destes atingem o ambiente, provenientes tanto de fontes naturais como também antropogênicas, por meio principalmente de descarte indevido de efluentes industriais e domésticos. Além destes milhares de compostos registrados, existem muitos outros que ainda não foram registrados ou simplesmente são desconhecidos, que também são lançados no ambiente. Somando-se a estes, é preciso ainda considerar os diversos metabólitos dessas substâncias e também aqueles formados a partir de reações entre compostos sintéticos e naturais. Dessa forma, é possível prever a gigantesca quantidade de compostos presentes no ambiente, especialmente nos corpos hídricos.

Menos de $0,001 \%$ desses compostos conhecidos e registrados são rotineiramente monitorados pelas agências governamentais como as dos Estados Unidos e Europa (VILLANUEVA et al., 2014; SCHMIDT et al., 2016). No Brasil, segundo a resolução 430/2011 do CONAMA (BRASIL, 2011) os efluentes de fontes poluidoras podem ser lançados diretamente no ambiente após tratamento adequado desde que as características desse efluente sigam os padrões de $\mathrm{pH}$, temperatura, presença de materiais sedimentáveis, vazão em que é lançado, presença de óleos e graxas, de materiais flutuantes e padrão de demanda bioquímica de oxigênio (BRASIL, 2011). Desta forma, se os efluentes não alterarem esses parâmetros, podem ser lançados sem restrições, ainda que contenham diversos tipos de substâncias tóxicas.

O tratamento convencional utilizado nas estações de tratamento de esgoto (ETEs) da Europa, por exemplo, consiste em dois tipos de processos, o físico e o biológico apenas, poucas vezes são usados tratamentos mais avançados como oxidação avançada e uso de ozônio, mas existe a busca por melhorias na remoção de micropoluentes orgânicos (ZORITA et al., 2009). Os tratamentos convencionais priorizam os parâmetros fisico-químicos, biológicos e a análise de alguns agentes químicos de importância e ocorrência no país ou região. Sendo assim, muitas das substâncias presentes em efluentes industriais, 
hospitalares e até mesmo domésticos, alguns desses micropoluentes orgânicos, não são retirados durante os tratamentos, principalmente os chamados contaminantes emergentes, que passaram a ser uma preocupação.

Contaminantes emergentes, em sua maioria, são identificados no ambiente em pequenas quantidades, em concentrações na ordem de $\mathrm{mg} / \mathrm{l}, \mu \mathrm{g} / \mathrm{L}$ ou até ng/L. Porém, é importante considerar que o composto não está presente no ambiente de forma isolada, junto desse estão diversos outros, podendo assim ocorrer interações, que podem modificar o efeito final observado em organismos não alvo (JONES et al., 2005; SCHMIDT et al., 2016).

Além de contaminantes emergentes como produtos de higiene, cosméticos e medicamentos, outros compostos a serem considerados são os metabólitos dos fármacos lançados através de efluentes domésticos e hospitalares. A excreção urinária é uma das principais vias de eliminação de medicamentos; uma parte do fármaco é excretada na forma original, inalterada, no entanto, na maioria dos casos, a maior porcentagem excretada é na forma biotransformada do fármaco (GURKE et al., 2015). Mostrando, assim, a importância de se considerar também os metabólitos de fármacos, principalmente os que possuem uma alta taxa de biotransformação.

Por meio da toxicologia regulatória é possível analisar o risco de compostos presentes no ambiente, e então estabelecer limites para a exposição segura de agentes químicos, seus metabólitos e também misturas complexas, e assim definir os padrões de qualidade hídricos (UMBUZEIRO et al., 2006). Antes da criação de leis e normas que estabelecem as condições de lançamento e os parâmetros para tratamento de efluentes, são necessários dados e estudos que indiquem o perigo e risco do composto, juntamente com balanço de fatores políticos e econômicos. Uma ferramenta usada na toxicologia regulatória é a avaliação de risco, que dentre seus objetivos estão: analisar o balanço entre o risco e o benefício de um agente químico, definir quais serão os possíveis alvos desse risco, estabelecer as prioridades para compor um projeto de atividades, fazer a estimativa do risco residual e da extensão da redução do risco mesmo após as medidas de redução de risco serem tomadas (FAUSTMAN, E.M.; OMENN, G.S., 2008).

A avaliação de risco pode ser dividida em quatro estágios: identificação do perigo, caracterização do perigo, avaliação da exposição e caracterização de 
risco. A primeira etapa tem por objetivo identificar qual o perigo que determinado agente químico possui, ou seja, determinar a toxicidade, prevendo eventos adversos causados por este, fazendo-se a análise da relação estruturaatividade, das propriedades físico-químicas, de estudos feitos em humanos e animais, bem como dos ensaios clínicos, epidemiológicos e toxicológicos. A caracterização do perigo relaciona dose e resposta do agente por meio de estudos cinéticos e dinâmicos. Na terceira etapa, o estudo leva em consideração a exposição dos organismos ao agente químico, sendo analisada o tipo de exposição em que a população será sujeita, a via de exposição, sua magnitude, duração, frequência e intensidade. Por fim, é feita a caracterização do risco, etapa em que é estudada a probabilidade de ocorrência de certo evento adverso, bem como a suscetibilidade da população que será provavelmente atingida e sua probabilidade de exposição (CORRÊA et al., 2014).

A avaliação de risco de diferentes compostos é de extrema importância para que se entenda qual o perigo e o risco relacionados à exposição desses, e para que assim sejam estabelecidas concentrações máximas para ocorrência no ambiente. Isso demonstra a relevância de estudos no contexto da toxicologia ambiental, pois esses contribuem não só para o conhecimento na área acadêmica e de pesquisa, mas também podem ser usados como base e fonte para estabelecimento de monitoramento ambiental, em que são regulamentadas as normas de qualidade do ambiente, além da tomada de decisão em casos específicos (MOERMOND et al., 2016).

\subsubsection{Presença de fármacos psicotrópicos no ambiente}

Nas últimas décadas os produtos farmacêuticos e cosméticos vem ganhando atenção no cenário ambiental pelos diversos relatos da ocorrência desses em águas superficiais, estações de esgoto e até mesmo em água potável, podendo assim considerá-los contaminantes emergentes de água (COMERO et al., 2013; NARBAITZ et al., 2013; SIMAZAKI et al., 2014; ZORITA et al., 2009; SILVEIRA et al., 2013).

Considerando o intenso uso, esses compostos são lançados na rede de esgoto e é justificável que tenham sido classificados como contaminantes ambientais emergentes, considerando ser esta a principal via de entrada de 
fármacos nos recursos hídricos (SUI et al., 2010; SILVA et al., 2015; YUAN et al., 2013). Diversos estudos têm mostrado a presença destes compostos em amostras ambientais. Os fármacos encontrados e suas quantidades dependem muito do perfil de tratamento empregado e de consumo de medicamentos mais utilizados nos diferentes países. Existem muitos relatos da presença de diversos fármacos psicotrópicos, dentre eles, os estudados neste trabalho, em águas superficiais e de tratamento como apresentado na Tabela 2. Esses quatro fármacos foram escolhidos devido a vasta presença no ambiente (Anexo A).

Tabela 2 - Ocorrência de fármacos psicotrópicos no ambiente em suas concentrações máximas e mínimas encontradas.

\begin{tabular}{|c|c|c|c|}
\hline Fármaco & $\begin{array}{l}\text { Concentração } \\
\text { encontrada }\end{array}$ & Local da coleta & Referência \\
\hline \multicolumn{4}{|l|}{ Venlafaxina } \\
\hline Máxima & $1,914 \mathrm{ug} / \mathrm{L}$ & $\begin{array}{l}\text { Esgoto hospitalar } \\
\text { não tratado, } \\
\text { Coimbra, } \\
\text { Portugal }\end{array}$ & $\begin{array}{l}\text { Santos et al., } \\
\quad 2013\end{array}$ \\
\hline Mínima & $0,2 \mathrm{ng} / \mathrm{L}$ & $\begin{array}{l}\text { Rios Vistula e } \\
\text { Ultrata; Polônia }\end{array}$ & $\begin{array}{c}\text { Giebultowicz; } \\
\text { Nałecz-Jawecki } \\
2014 \\
\end{array}$ \\
\hline \multicolumn{4}{|l|}{ Haloperidol } \\
\hline Máxima & $2,69 \mu \mathrm{g} / \mathrm{L}$ & $\begin{array}{c}\text { Máxima } \\
\text { encontrada em } \\
\text { amostras da } \\
\text { União Européia }\end{array}$ & Loos et al., 2013 \\
\hline Mínima & $0,24 \mathrm{ng} / \mathrm{L}$ & $\begin{array}{l}\text { Águas } \\
\text { superficiais, } \\
\text { Suécia }\end{array}$ & Fick et al., 2011 \\
\hline \multicolumn{4}{|c|}{ Carbamazepina } \\
\hline Máxima & $27,7 \mu \mathrm{g} / \mathrm{L}$ & $\begin{array}{l}\text { Água residual de } \\
\text { estação de } \\
\text { tratamento de } \\
\text { esgoto, Chipre }\end{array}$ & $\begin{array}{l}\text { Fatta-Kassinos, } \\
\text { Hapeshi, et al. } \\
2011\end{array}$ \\
\hline Mínima & $0,08 \mathrm{ng} / \mathrm{L}$ & $\begin{array}{l}\text { Rio Lobregat, } \\
\text { Espanha }\end{array}$ & $\begin{array}{c}\text { Ginebreda et al., } \\
2010\end{array}$ \\
\hline \multicolumn{4}{|l|}{ Fluoxetina } \\
\hline Máxima & 9676 ng/L & $\begin{array}{l}\text { Influente bruto } \\
\text { antes da } \\
\text { sedimentação } \\
\text { primária, Portugal }\end{array}$ & $\begin{array}{l}\text { Salgado et al., } \\
2011\end{array}$ \\
\hline
\end{tabular}




\begin{tabular}{|c|c|c|c|}
\hline Mínima & $0,04 \mathrm{ng} / \mathrm{L}$ & $\begin{array}{c}\text { Estimativa } \\
\text { concentração } \\
\text { ambiental } \\
\text { nacional, } \\
\text { Dinamarca }\end{array}$ & $\begin{array}{l}\text { Styrishave et al., } \\
2011\end{array}$ \\
\hline
\end{tabular}

No Brasil, estudos realizados por Silva e colaboradores (2015) na costa norte de Salvador, Bahia, identificaram medicamentos psicotrópicos presentes em quantidades significantes nas amostras de sedimentos coletados na Baia de todos os Santos, que é o destino de água residuais de residências e hospitais da região.

Como já citado, os contaminantes emergentes são encontrados em amostras ambientais em concentrações muito baixas, na ordem de $\mu \mathrm{g} / \mathrm{L} \mathrm{e} \mathrm{ng} / \mathrm{L}$, porém, vale aqui lembrar que os fármacos psicotrópicos são desenvolvidos para atuar em sistemas biológicos em doses baixas (ANKLEY et al., 2007; ROOS et al., 2012), dessa forma, mesmo nessas concentrações ambientais é possível que esses compostos provoquem algum tipo de efeito em diferentes organismos não alvo.

Evidentemente, uma maior concentração de fármacos é encontrada em efluentes domésticos e hospitalares, que são lançados em estações de tratamento de esgoto municipais antes de serem descartados em corpos d'água. Porém, muitos dos fármacos são apenas parcialmente removidos, pois são resistentes ao tratamento convencional utilizado (PALMER et al., 2008; SILVA et al., 2011; DE ALMEIDA et al., 2013). Por consequência, ainda são encontradas pequenas concentrações de fármacos e seus metabólitos tanto em água potável como em águas residuais e rios. Gurke et al., (2015) verificaram a ocorrência e a remoção de medicamentos frequentemente prescritos na Alemanha; dos 45 compostos estudados, 39 obtiveram uma taxa de remoção abaixo de $50 \%$ após passar pela estação de tratamento de esgoto, e para apenas um deles foi observada eficiência superior a $80 \%$ de remoção. Tendo em vista a presença desses fármacos em águas potáveis, é preciso considerar que essa contaminação ambiental atinge não só a fauna e flora, mas também os humanos, de forma direta ou indireta. 
Yuan et. al., (2013) estudaram 22 medicamentos psicotrópicos em amostras de efluentes de ETEs na China, incluindo venlafaxina e haloperidol, objetos de estudo deste trabalho. Os autores concluíram que o tratamento primário nas ETEs não é eficiente para esta classe de medicamentos, removendo no máximo $60 \%$, devido à alta resistência a degradação, tanto abiótica como biótica.

A difícil remoção dos fármacos se deve principalmente às características físico-químicas. A fluoxetina, por exemplo, tem alto poder de adsorção aos lodos, sendo assim dispersada tanto em resíduos de água, como lodo das ETEs (KWON et al., 2006; REDSHAW et al., 2008). Além disso, esse medicamento apresenta alta estabilidade hidrolítica e fotolítica, sendo considerado um dos mais persistentes no meio ambiente (CALISTO; ESTEVES, 2009).

Como muitos medicamentos são resistentes aos tratamentos convencionais de água, sendo apenas parcialmente removidos (PALMER et al., 2008; SILVA et al., 2011), a exposição de organismos aquáticos a estes compostos é esperada. Ainda que sejam encontradas pequenas concentrações destes compostos em amostras de água após o tratamento (na ordem de ng/L ou até $\mu \mathrm{g} / \mathrm{L}$ ), as quantidades encontradas são capazes de causar efeito, sendo dessa forma classificados como micropoluentes (LUO et al., 2014). Adicionalmente, é preciso considerar que a exposição a esses compostos acontece no ambiente de forma crônica, podendo assim haver acumulação desses no organismo.

Cerca de $15 \%$ dos medicamentos utilizados em hospitais têm potencial de bioacumulação (JEAN et al., 2012). Um estudo realizado por Brooks et al., (2005) quantificou diversos medicamentos em tecidos de peixes, dentre eles a fluoxetina. Foi encontrado 1,58 ng/g de fluoxetina no cérebro, 1,34 ng/g no fígado e $0,11 \mathrm{ng} / \mathrm{g}$ em tecidos musculares de peixes. Visto isso, os medicamentos presentes no ambiente expõem não apenas o ecossistema aquático, mas também os humanos, que poderão entrar em contato não só por meio da ingestão de água, mas também através da alimentação de peixes que acumularam esses fármacos em seus tecidos.

Assim, estudos e ensaios toxicológicos devem ser feitos com as substâncias presentes nestas amostras de águas residuais para que se entendam os possíveis danos e impactos desses agentes nos organismos alvo 
e não alvo, como os peixes e humanos. Além de estudos com as substâncias na forma isolada, é preciso considerar que no ambiente aquático essas estarão presentes juntamente com diversas outras, sendo importante assim analisar além da ação independente dos compostos, a mistura desses (SCHMIDT et al., 2016) que pode potencializar ou antagonizar os efeitos tóxicos dos compostos.

Todavia, os estudos ecotoxicológicos abordando os contaminantes emergentes, como os fármacos psicotrópicos, mesmo que de forma isolada, ainda são bastante escassos. No Brasil e outros países em desenvolvimento, as análises ambientais para verificação da presença de fármacos vêm crescendo, mas ainda são poucas, ao contrário de países desenvolvidos como Estados Unidos, países da Europa e Japão (BEEK et al., 2016). Além das análises químicas, é de fundamental importância entender qual é o perigo e quais são os riscos da exposição tanto ambiental como humana, para que assim, a contaminação de efluente e corpos hídricos com esses compostos tenha a devida atenção dos órgãos responsáveis. 


\subsection{Avaliação de toxicidade ambiental}

Como já citado, tendo em vista a resistência de diversos medicamentos aos tratamentos convencionais de águas residuais, é bem provável que resíduos desses atinjam o ambiente aquático, impactando não só a qualidade da água de rios e cursos de água, mas também a população aquática, como os peixes.

Uma importante etapa da avaliação de risco ambiental é a análise de toxicidade aquática e bioacumulação de um agente químico específico ou da amostra. Com essas análises são observados efeitos em organismos aquáticos de três níveis tróficos: um representante das plantas (normalmente as algas), um de invertebrados (como os crustáceos), e um representante dos animais vertebrados (sendo o peixe o mais usado) (HALDER et al., EURL ECVAM, 2014). $\mathrm{Na}$ avaliação da toxicidade aquática são feitos inicialmente os testes de toxicidade aguda, e se indicado risco ou quando se prevê uma exposição prolongada, os testes de toxicidade crônica são realizados posteriormente (HALDER et al., EURAL ECVAM, 2014). Nos ensaios de toxicidade aguda, comumente são usados peixes, pois são bons representantes do meio aquático, com um papel importante na cadeia alimentar, sendo um dos últimos animais na cadeia aquática, além de serem fonte de alimentação para diversos animais superiores e para humanos. Sendo assim, os peixes são também muito usados como animais sentinelas para a qualidade de águas (LAMMER et al., 2009). A avaliação de toxicidade aguda para peixes é exigida na União Europeia para a liberação de diversos compostos como químicos industriais, produtos biocidas, medicamentos veterinários, aditivos alimentares (HALDER, et al, EURAL ECVAM, 2014), e também para águas residuais na Alemanha (ALEMANHA, 1998).

Visando desenvolver um método alternativo ao uso de animais, estudos coordenados pelo laboratório europeu referência de testes alternativos ao uso de animais, o ECVAM (Centro Europeu para Validação de Métodos Alternativos, do inglês: European Centre for the Validation of Alternative Methods) juntamente com a OECD (Organização para a Cooperação e Desenvolvimento Econômico, do inglês: Organization for Economic Co-operation and Development), em 2009, validou o teste de toxicidade aguda com embriões de zebrafish, para que houvesse uma redução no número de peixes adultos usados em testes 
(EUROPEAN COMISSION, EURL ECVAM, 2014). O ensaio com embriões não é tido pela União Europeia como um teste in vivo, visto que segundo a Diretriz 2010/63/EU que visa a proteção animal para usos científicos, são considerados in vitro testes que utilizam organismos que ainda não adquiriram a capacidade de alimentação de forma independente (UNIÃO EUROPEIA, 2010). Paralelamente, em 2012, Belanger e colaboradores compararam testes de toxicidade aguda utilizando peixes adultos e embriões de zebrafish, resultando em uma forte correlação entre os ensaios (BELANGER et al., 2012), comprovando assim a eficiência e correspondência dos ensaios com embriões.

Antes mesmo da validação do método, a Alemanha já utilizava o teste com embriões de zebrafish para avaliação de toxicidade aguda em amostras de águas residuais, regulamentado pela Lei Gazette desde 2005 (ALEMANHA, 2005). Em 2007, já havia sido feita a padronização, pela organização internacional de padronização, a ISO 15088 (Organização Internacional para Padronização, do inglês: International Organization for Standardization) da determinação de toxicidade aguda de águas utilizando-se embriões (ISO 15088, 2007).

Frente à grande atenção dos órgãos ao uso de métodos alternativos a animais, visando maior respeito ao bem-estar animal e redução do uso de animais para fins científicos, a substituição de peixe adulto por estágios embrionários deve sempre ser feita quando possível e apropriado (UNIÃO EUROPEIA, 2014), conseguindo atender assim aos princípios de redução, refinamento e substituição, conhecidos como princípio dos 3Rs (reduction, refinement, replacement) publicados em 1959 por William Russel e Rex Burch (HUNTINGFORD et al., 2006; LAMMER et al 2009b; RUSSEL; BURCH, 1959). Sendo assim, os ensaios de toxicidade aguda ambiental com estágios embrionários de zebrafish vem ganhando cada vez mais espaço.

\subsubsection{Utilização de embriões de zebrafish como modelos de toxicidade ambiental}

Como explanado anteriormente, testes de toxicidade aguda utilizando embriões de peixes são uma alternativa aos testes com adultos. Dentro deste contexto, a espécie Danio rerio vem ganhando cada vez mais destaque no 
cenário científico nacional e internacional. Trata-se de uma espécie de peixe de porte pequeno, medindo em média $40 \mathrm{~mm}$ de comprimento, da família Cyprinidae, possui o formato do corpo longilíneo fusiforme e achatado nas laterais, onde possui de 5 a 7 linhas longitudinais características em tons azulados (SPENCE et al., 2008), que os deixou conhecido como Zebrafish (Figura 1).

Figura 1. Imagem do peixe zebrafish adulto, sendo o peixe da frente fêmea, e os dois no plano atrás machos (Fonte: Autor).

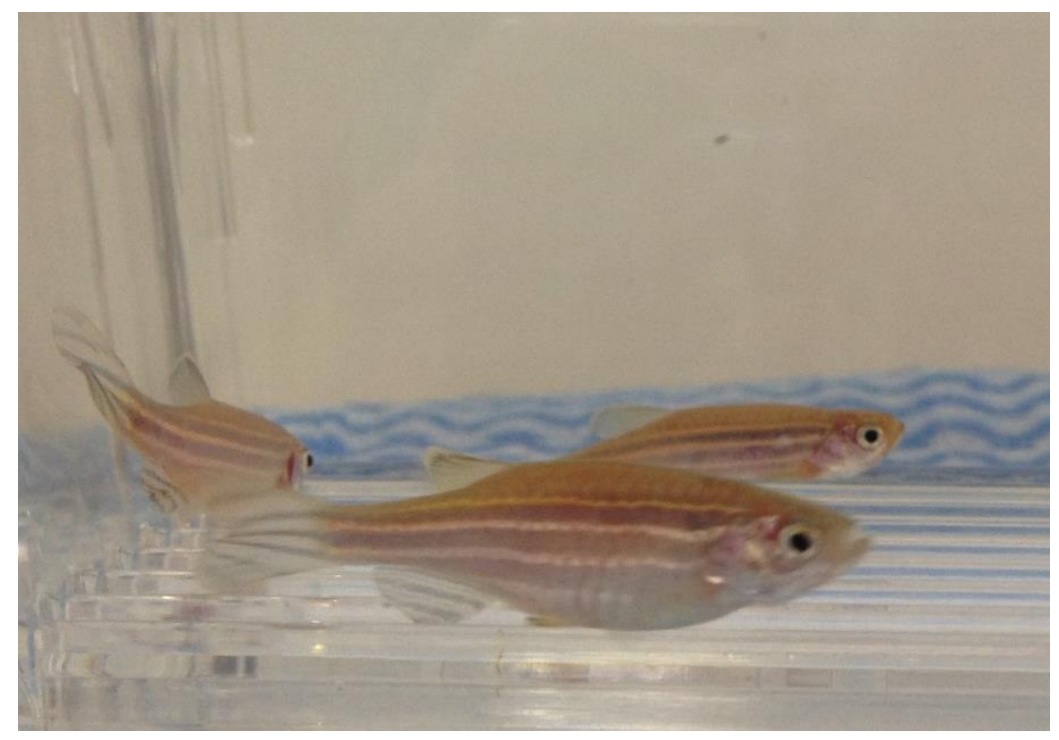

Zebrafish é uma espécie de peixe de fácil manutenção, com desenvolvimento bem definido e rápido (TEIXIDÓ et al.,2012), possuindo não só o benefício de poder utilizar o estágio embrionário, reduzindo o conflito ético de causar incômodo e sofrimento aos animais, mas também a vantagem de que os embriões são mais sensíveis, respondendo melhor e mais rapidamente quando comparado aos adultos (NAGEL, 2002).

Embrião é o intervalo de vida entre a fertilização até a eclosão do ovo (aproximadamente 72 horas pós fertilização - hpf). A continuidade do desenvolvimento do embrião ocorre fora dos limites do ovo durante o intervalo conhecido como estágio larval (LAMMER et al 2009a).

É um modelo experimental que une as vantagens de experimento in vitro com as vantagens do in vivo, tem a maior praticidade, resultados em tempos menores e custos mais baixos condizentes com testes in vitro, mas com a 
complexidade de um organismo inteiro, possuindo metabolismo próprio e correlação entre diferentes células e tecidos (TRUONG et al., 2011). Além disso, a espécie de peixe Danio rerio possui uma similaridade genômica com os humanos, cerca de $70 \%$ dos genes da espécie tem ao menos um ortólogo ao do humano, sendo o contrário também verdadeiro. Esses genes são relacionados com fatores de transcrição de proteínas e com atividade neural, dessa forma 0 Danio rerio é bastante usado em diversos estudos como um modelo genômico (HOWE et al., 2013), bem como em ensaios de avaliação de neurotoxicidade de compostos químicos.

$\mathrm{Na}$ análise dos estágios embrionários e larvais do zebrafish são feitos estudos dos efeitos de teratogenicidade, devido ao desenvolvimento do embrião ser fora do corpo materno e de o córion do embrião e corpo da larva serem transparentes (NAGEL, 2002), facilitando o acompanhamento do desenvolvimento, possibilitando a identificação de malformações durante a fase embrio-larval, sem a necessidade de sacrificar o organismo.

A organogênese completa do Danio rerio se dá de forma rápida, ao final de 6 dias, por exemplo, já é possível observar estruturas importantes como coração, bexiga natatória, sistema circulatório e formato esquelético (KIMMEL et al., 1995). Considerando essas vantagens, o modelo experimental com embriões de zebrafish vem ganhando cada vez mais espaço, sendo um dos organismos padrões que mais cresce em publicações científicas (GARCIA et al., 2016). Além de ser usado para avaliações dentro da toxicologia ambiental, os experimentos com embriões de zebrafish têm sido empregados para ensaios de segurança e toxicidade de produtos nutracêuticos, estudos relacionados a diversas doenças, como doença de Parkinson, distúrbios renais e doenças cardiovasculares, além de ser também uma ótima alternativa para análises na fase de screening de novos medicamentos, visando investigação de possível ação teratogênica (BIAN et al., 2016; BRETAUD et al., 2004; DOOLEY,K.; ZON, L., 2000; GIACOMINI et al., 2006). Um estudo publicado em 2014 por Yamashita e colaboradores, analisou 59 compostos que são classificados como teratogênicos e não teratogênicos em modelos in vivo usando mamíferos, a concordância de teratogenicidade positiva foi de $88 \%$ e de não-teratogenicidade foi de $93 \%$, indicando assim uma grande relação de correspondência entre os estudos feitos com mamíferos e com embriões de zebrafish. 
O sistema nervoso do zebrafish também é bem desenvolvido nos primeiros dias de vida, neurônios dopaminérgicos e colinérgicos podem ser detectados nos estágios iniciais de desenvolvimento (GIACOMINI et al., 2006; BEHRA et al., 2002). Além disso, é possível observar também padrões comportamentais em poucos dias após a fertilização, por exemplo, pode-se notar atividades como fuga, busca por alimento, perfil de nado e movimentação, ainda no estágio larval (GIACOMINI et al., 2006). Assim, o padrão de locomoção e atividade do embrião de zebrafish tem sido muito usado para relacionar e avaliar o desenvolvimento de neurotoxicidade (SELDERSLAGHS et al., 2010).

Outra grande vantagem que fez com que esta espécie se destacasse em testes neurocomportamentais é que a estrutura básica do sistema nervoso em peixes apresenta semelhanças com a de mamíferos e os mesmos neurotransmissores são encontrados (PANULA et al., 2010). Assim, visando complementar a avaliação da neurotoxicidade, propomos também a utilização de um biomarcador deste efeito, a atividade da enzima colinesterase. Esta enzima cliva rapidamente a acetilcolina em colina e acetato e é amplamente distribuída no reino animal, comum aos humanos e Danio rerio, e crítica para a transmissão sináptica entre neurônios tanto em sinapses colinérgicas quanto entre junções neuromusculares. Estas atividades configuram a base de muitas das funções biológicas essenciais, tais como o batimento cardíaco, respiração, digestão e a atividade cerebral (FISCHER et al., 2015). Um dos efeitos causados por xenobióticos consiste em mudanças na sinalização colinérgica que conduzem a um desequilíbrio nos níveis de acetilcolina extracelular. A inibição da acetilcolinesterase (AChE, do inglês: acetylcholinesterase) resulta na hiperestimulação dos receptores de acetilcolina pós-sinápticos, o que leva a aberrações fisiológicas que vão desde a disfunção comportamental à morte (HAVERROTH et al., 2015). A atividade desta enzima é um clássico biomarcador de efeito de praguicidas pertencentes à classe dos carbamatos $\mathrm{e}$ organofosforados, no entanto, a especificidade para praguicidas vem sendo questionada. Já em 1998, Guilhermino et al., mostraram que metais (molibdênio, bário e crômio) e detergentes (dodecilbenzilsulfonato e dodecil sulfato de sódio) também induzem à redução da atividade desta enzima em mexilhões. Seguindo a mesma tendência, em 2010, Frasco et al., utilizando ensaios in vitro com AChE isolada sugerem que a atividade da enzima deva ser utilizada como biomarcador 
de neurotoxicidade para metais, com exceção do níquel. Similarmente, a atividade da enzima AChE tem sido considerada um bom biomarcador de neurotoxicidade em peixes expostos a hidrocarbonetos policíclicos aromáticos (ALMEIDA et al., 2012; SILVA et al., 2013). Adicionalmente, a atividade da enzima colinesterase tem sido sugerida como um dos biomarcadores usados na avaliação de neurotoxicidade de peixes marinhos expostos a misturas complexas de poluentes (GRAVATO et al., 2010).

A grande vantagem da utilização do ensaio com embriões de Danio rerio é o fato de que os demais vertebrados possuem dois tipos de enzimas colinesterases, a acetilcolinesterase e a butirilcolinesterase, já essa espécie de peixe possui genes para a expressão apenas da AChE, tornando-se assim um ótimo modelo para análise da atividade da enzima específica (RODRIGUEZFUENTES et al., 2015; BERTRAND et al., 2004).

Juntamente à atividade da $\mathrm{AChE}$, um outro parâmetro muito analisado utilizando embriões de zebrafish é a atividade e movimentação desses frente à exposição a toxicantes e amostras aquáticas, visto que um comportamento inadequado pode ter como impacto a sobrevivência desses animais (SCOTT et al., 2004), podendo influenciar não apenas esses, mas também toda o ecossistema vinculado a eles, como predadores e fontes de alimento. O perfil comportamental de peixes é o resultado de uma junção de diferentes sistemas, como o sensorial, hormonal, metabólico e neurológico (SCOTT et al., 2004), sendo possível dessa forma relacionar comportamento com atividades do organismo.

Dessa maneira, esse trabalho tem como propósito avaliar os efeitos de medicamentos psicotrópicos presentes no ambiente sobre o desenvolvimento embrio-larval de zebrafish, avaliando a indução de possíveis malformações, além de modificações neurocomportamentais, tanto dos fármacos isolados, quanto em misturas. Isto posto, o trabalho é parte inicial de uma avaliação de risco, analisando-se o perigo desses compostos. 


\section{CONCLUSÕES}

A partir dos resultados obtidos neste trabalho, através da avaliação dos três níveis de organização biológica usando estágios iniciais de zebrafish, é possível concluir que nas condições dos tratamentos:

- Os quatro fármacos são estáveis durante os 6 dias, nas condições dos testes realizados;

- Tanto a mistura como os quatro fármacos individualmente não apresentaram efeitos teratogênicos significativos em embriões de zebrafish até $144 \mathrm{hpf}$ expostos à concentrações ambientalmente relevantes;

- Os quatro fármacos de forma individual e a mistura desses não causam alteração significativas na movimentação de embriões de 28hpf expostos às concentrações ambientalmente relevantes;

- Os fármacos venlafaxina e carbamazepina não provocaram alteração significativa na atividade da enzima AChE em embriões de 96hpf expostos às concentrações ambientalmente relevantes;

- O haloperidol aumentou a atividade enzimática dos embriões em concentrações muito baixas, com posterior retorno aos níveis basais em doses mais altas;

- A fluoxetina aumentou a atividade da enzima AChE dos embriões de zebrafish na maior concentração testada, ainda que esta seja considerada baixa;

- Esse efeito de aumento na atividade enzimática foi antagonizado na mistura dos fármacos.

\subsection{Considerações Finais}

Este trabalho mostra que mesmo concentrações muito baixas, na ordem de $\mathrm{ng} / \mathrm{L}$, podem causar algum efeito em organismos. Assim, é necessário que sejam testadas não apenas as concentrações próximas ao LOAEL ou IC50 dos compostos químicos, devido à possibilidade de manifestação do fenômeno hormesis. 
Para a confirmação da hormesis nos fármacos haloperidol e fluoxetina ainda são necessários mais ensaios, para entender a partir de qual concentração a atividade enzimática começa a diminuir para chegar nos valores basais e, quando inicia a inibição da atividade em embriões com a mesma idade. Assim, seria possível construir uma curva dose-resposta, confirmando a hipótese da característica bifásica.

Nosso trabalho mostra importância de estudos conduzidos com concentrações ambientalmente relevantes, para que seja possível uma correta avaliação do risco do lançamento destes compostos no ambiente aquático, bem como a avaliar a necessidade de outros tratamentos de efluentes. 


\section{REFERÊNCIAS}

AL-RIFAI, J.H.; KHABBAZ, H.; SCHAFER. Removal of pharmaceuticals and endocrine disrupting compounds in a water recycling process using reverse osmosis systems. Separation and Purification Technology, v. 77, p. 60-67, 2011.

ALEMANHA. Federal Law Gazette I. Act Pertaining to Charges Levied for Discharging Waste Water into Waters. 25 August 1998.

ALFENA, M.D. Uso de psicotrópicos na atenção primária. 2015. 68f. Dissertação (Mestrado Modalidade Profissional em Saúde Pública) - Escola Nacional De Saúde Pública - Fiocruz, Rio de Janeiro, 2015

ALMEIDA, J.R.; GRAVATO, C.; GUILHERMINO,L. Challenges in assessing the toxic effects of polycyclic aromatic hydrocarbons to marine organisms: A case study on the acute toxicity of pyrene to the European seabass (Dicentrarchus labrax L.). Chemosphere, v. 88, p. 926-937, 2012.

ALONSO, S.G.; CATALÁ, M.; MAROTO, R.R.; GIL, J.L.R.; MIGUEL, A.G.; VALCÁRCEL, Y. Pollution by psychoactive pharmaceuticals in the Rivers of Madrid metropolitan area (Spain). Environment International, v. 36, n. 2, p. 195201, 2010.

ANKLEY, G.T.; BROOKS, B.W.; HUGGETT, D.B.; SUMPTER, J.P. Repeating history: pharmaceuticals in the environment. Environmental Science and Technology, v. 41, p. 8211-8217, 2007.

BAKKERS, J. Zebrafish as a model to study cardiac development and human cardiac disease. Cardiovascular Research, v. 91, p. 279-288, 2011.

BARBAZUK, W.B.; KORF, I.; KADAVI, C.; HEYEN, J.; TATE, S.; WUN, E. BEDELL, J.A.; MCPHERSON, J.D.; JOHNSON, S.L. The Syntenic Relationship of the Zebrafish and Human Genomes. Genome Research, v. 10,p. 1351-135, 2000.

BEEK,T.; WEBER, F.A.; BERGMANN, A.; HICKMANN, S.; EBERT, I.; HEIN, A.; KÜSTER, A. Pharmaceuticals in the environment-global occurrences and perspectives. Environmental Toxicology and Chemistry, v. 9999, n. 9999, p. 1-13, 2016.

BEEKHUIJZEN, M.; DE KONING, C.; FLORES-GUILLÉN, M.-E.; DE VRIESBUITENWEG, S.; TOBOR-KAPLON, M.; VAN DE WAART, B.; EMMEN, H. From cutting edge to guideline: $A$ first step in harmonization of the zebrafish embryotoxicity test (ZET) by describing the most optimal test conditions and morphology scoring system. Reproductive toxicology (Elmsford, New York), v. 56, p. 64-76, 15 ago. 2015.

BEHRA, M.; COUSIN, X.; BERTRAND, C.; VONESCH, J.L.; BIELLMANN, D; CHATONNET A.; STRAHLE, U. Acetylcholinesterase is required for neuronal 
and muscular development in the zebrafish embryo. Nature neuroscience, v. 5 , n. 2, p. 111-118, 2002.

BELANGER, S.E.; RAWLINGS, J.M.; CARR, G.C. An Update to the Fish Embryo Toxicity-Acute Fish Toxicity Relationship and Prospects for Support of the Use of the FET as an Animal Alternative. Document Developed for the February, 2012 Meeting of the OECD ad hoc Expert Group on the Fish Embryo Test. 22 Fev. 2012.

BERTRAND, C.; CHATONNET, A.; TAKKE, C.; YAN, Y.; POSTLETHWAIT, J.; TOUTANT, J.P.; COUSIN, X. Zebrafish Acetylcholinesterase Is Encoded by a Single Gene Localized on Linkage Group 7. The Journal Of Biological Chemistry, v. 276, n. 1, p. 464-474, 2001.

BIAN W.P.; PEI, D.S. Zebrafish model for safety and toxicity testing of nutraceuticals. In: GUPTA, R.C. (Org). Nutraceuticals: Efficacy, Safety and Toxicity. San Diego, USA: Elsevier Science and Technology, 2016.

BLYTHE, D.; HACKETT, L.P. Cardiovascular and neurological toxicity of venlafaxine. Human and Experimental Toxicology, v. 18, n. 5, p. 309-313, 1999.

BOUISSOU-SCHURTZ, C.; HOUETO, P.; GUERBET, M.; BACHELOT, М.; CASELLAS, C.; MAUCLAIRE, A.C.; PANETIER, P.; DELVAL, C.; MASSET,D. Ecological risk assessment of the presence of pharmaceutical residues in a French national water survey. Regulatory Toxicology and Pharmacology, v. 69, p. 296-303, 2014.

BRASIL. Ministério da Saúde. Agência Nacional de Vigilância Sanitária. Sistema De Acompanhamento do Mercado de Medicamentos. Mercado Farmacêutico Brasileiro 2015. Brasília, DF, 2016.

BRASIL. Ministério da Saúde. Agência Nacional de Vigilância Sanitária. Resolução RDC N 49, de 14 de Novembro de 2015. Brasília, DF, 2015.

BRASIL. Conselho Nacional do Meio Ambiente. Resolução CONAMA N 430. Brasília, DF, 13 Maio. 2011.

BRASIL. Previdência Social. Acompanhamento Mensal dos Benefícios Auxílios-Doença Previdenciários, Concedidos Segundo os Códigos da CID-10 Janeiro a Dezembro de 2016. Brasília, DF, 30 jan. 2017.

BRASIL. Ministério da Saúde. Agência Nacional de Vigilância Sanitária. Resolução RE $n^{\circ} 899$ de 29 de maio de 2003. Dispõe sobre a Validação de Métodos Analíticos e Bioanalíticos. Brasília, DF, 02 jun. 2003.

BRETAUD, S.; LEE, S.; GUO, S. Sensitivity of zebrafish to environmental toxins implicated in Parkinson's disease. Neurotoxicology and Teratology, v. 26, p. 857-864, 2004. 
BROOKS, B.W.; CHAMBLISS, C.K.; STANLEY, J.K.; RAMIREZ, A.; BANKS, K.E.; JOHNSON, R.D.; LEWIS, R.J. Determination of select antidepressants in fish from an effluent-dominated stream. Environmental toxicology and chemistry, v. 24, n. 2, p. 464-469, 2005.

BRUNTON, L.L.; CHABNER, B.A.; KNOLLMAN, B.C. As Bases Farmacológicas da Terapêutica. 12 ${ }^{\text {a }}$. ed. Porto Alegre: AMGH Editora, 2012. CAS, Chemical Abstracts Service, CAS Registry. Disponível em: < www.cas.org >. Acesso em 01 de Abril de 2017.

CALABRESE, E.J. Neuroscience and Hormesis: Overview and General Findings. Critical Reviews in Toxicology, v. 38, p.249-252, 2008.

CALABRESE, E.J. Hormesis: why it is important to toxicology and toxicologists. Environmental Toxicology and Chemistry, v. 27, n. 7, p. 1451-1474, 2008.

CALABRESE, E.J.; BALDWIN, L.A. Toxicology rethinks its central belief. Nature, v. 421, p. 691-692, 2003.

CALABRESE, E.J.; BALDWIN, L.A. Defining hormesis. Human \& Experimental Toxicology, v. 21, p. 91-97, 2002.

CALABRESE, E.J.; BLAIN, R.B. The hormesis database: The occurrence of hormetic dose responses in the toxicological literature. Regulatory Toxicology and Pharmacology, v. 61, p. 73-81, 2011.

CALISTO, V.; ESTEVES, V. I. Psychiatric pharmaceuticals in the environment. Chemosphere, v. 77, n. 10, p. 1257-1274, Nov. 2009.

CARLINI, E.A.; NOTO, A.R.; NAPPO, S.A.; SANCHEZ, Z.M.; FRANCO, V.L.S.; SILVA, L.C.F.; SANTOS, ALVES, D.C. Fluoxetina: indícios de uso inadequado. Jornal Brasileiro de Psiquiatria, n. 58, v. 2, p. 97-100, 2009.

CCHR, Citizens Commission on Human Rights, Total number of people taking psychiatric drugs in the United States. Disponível em: < https://www.cchrint.org/psychiatric-drugs/people-taking-psychiatric-drugs/>.

Acesso em 28 de Março de 2017.

CHAPMAN, P.M. The implications of hormesis to ecotoxicology and ecological risk assessment. Human \& Experimental Toxicology, v. 20, p. 499 -505, 2001.

CHEN, Y.H.; HUANG,Y.H.; WEN,C.C.; WANG, Y.H.; LICHEN, W.; CHEN,L.C.; TSAY,H.J. Movement disorder and neuromuscular change in zebrafish embryos after exposure to caffeine. Neurotoxicology and Teratology, v. 30, n. 5, p. 440447, 2008.

CHOI, V.W.Y.; CHEUNG, A.L.Y. CHENG, S.H.; YU, K.N. Hormetic Effect Induced by Alpha-Particle-Induced Stress Communicated In Vivo between Zebrafish Embryos. Environmental Science \& Technology, v. 46, p. 11678-11683, 2012. 
COMERO, S.; LOOS, R.; CARVALHO, R.; ANTO, D.C.; LOCORO, G.; TAVAZZE, S.; PARACCHINI, B.; GHIANI, M.; LETTIERI, T.; BLAHA, L.; JAROSOVA, B.; VOORSPOELS, S.; SERVAES, K.; HAGLUND, P.; FICK, J.; LINDBERG, R.H.; SCHWEISIG, D.; GAWLINK, B.M. EU-wide monitoring survey on emerging polar organic contaminants in wastewater treatment plant effluents. Water Research, v. 4 7, p. 6475-6487, 2013.

CORRÊA, C.L.; ALONZO, H.G.A.; TREVISAN, R.M.S. Avaliação de Risco. In: OGA, S.; CAMARGO, M.M. DE A.; BATISTUZZO, J. A. DE O. (Org.). Fundamentos de Toxicologia. 4a . ed. São Paulo: Atheneu Editora, 2014.

DE ALMEIDA, C.A.A.; BRENNER, C.G.B.; MINETTO, L.; MALLBANN, C.; MARTINS, A. Determination of anti-anxiety and anti-epileptic drugs in hospital effluent and a preliminary risk assessment. Chemosphere, v. 93, n. 10, p. 23492355, 2013.

DOOLEY, K.; ZON, L.I. Zebrafish: a model system for the study of human disease. Current Opinion in Genetics and Development, v. 10, n. 3, p. 252256, 2000.

EARNEST, D.J.; TUREK, F.W. Role for acetylcholine in mediating effects of light on reproduction. Science, v. 219, p. 77-79, 1983.

ELLMAN, G.L.; COURTNEY, K.D.; ANDRES, V.; FEATHERSTONE, R.M. A new and rapid colorimetric determination of acetylcholinesterase activity. Biochemical Pharmacology, v. 7, n. 2, p. 88-95, 1961.

EUROPEAN COMISSION. EURL ECVAM Recommendation on the Zebrafish Embryo Acute Toxicity Test Method (ZFET) for Acute Aquatic Toxicity Testing. JCR Science and Policy Reports. Jul 2014.

FATTA-KASSINOS, D.; HAPESHI, E.; ACHILLEOS, A.; MERIC, S.; GROS,M.; PETROVIC, M.; BARCELO, D. Existence of Pharmaceutical Compounds in Tertiary Treated Urban Wastewater that is Utilized for Reuse Applications. Water Resources Manage, v. 25, p. 1183-1193, 2011.

FAUSTMAN, E.M.; OMENN, G.S. Risk Assessment. In: KLASSEN, C.D. Casarett \& Doull's Toxicology: The Basic Science of Poisons. $7^{\text {th }}$. ed. New York: McGraw-Hill, 2008.

FERREIRA, P.G.; COSTA, S.; DIAS, N.; FERREIRA, A.J.; FRANCO, F. Simultaneous interstitial pneumonitis and cardiomyopathy induced by venlafaxine. Jornal Brasileiro de Pneumologia, v. 40, p. 313-318, 2014.

FERRER, I.; THURMAN, E.M. Analysis of 100 pharmaceuticals and their degradates in water samples by liquid chromatography/quadrupole time-of-flight mass spectrometry. Journal of Chromatography A, v. 1259, p. 148-157, 2012.

FICK, J.; LINDBERG, R.H.; KAJ, L.; BRORSTRÖM-LUNDÉN, E. Results from the Swedish National Screening Programme 2010 Subreport 3. Pharmaceuticals, 
IVL Swedish Environmental Research Institute Ltd. 2011.

FISCHER, A.; WOLMAN, M.; GRANATO, M.; PARSONS, M.; MCCALLION, A. S.; PROESCHER, J.; ENGLISH, E. Carbamate nerve agent prophylatics exhibit distinct toxicological effects in the zebrafish embryo model. Neurotoxicology and Teratology, v. 50, p. 1-10, 2015.

FRASCO, M.F.; FOURNIER, D.; CARVALHO, F.; GUILHERMINO, L. Do metals inhibit acetylcholinesterase (AChE)? Implementation of assay conditions for the use of AChE activity as a biomarker of metal toxicity. Biomarkers, v. 10, p 360$375,2010$.

GARCIA, G.R.; NOYES, P.D.; TANGUAY, R.L. Advancements in zebrafish applications for 21 st century toxicology. Pharmacology and Therapeutics, $v$. 161, p. 11-21, 2016.

GIACOMINI, N.J.; ROSE, B.; KOBAYASHI, K.; GUO, S. Antipsychotics produce locomotor impairment in larval zebrafish. Neurotoxicology and Teratology, v. 28, n. 2, p. 245-250, 2006.

GIEBULTOWICZ, J.; NALECZ-JAWECKI, G. Occurrence of antidepressant residues in the sewage-impacted Vistula and Utrata rivers and in tap water in Warsaw (Poland). Ecotoxicology and Environmental Safety, v. 104, n. 1, p. 103-109, 2014.

GINEBREDA, A.; MUNOZ, I.; ALDA, M.L.; BRIX, R.; LÓPEZ-DOVAL, J.; BARCELÓ, D. Environmental risk assessment of pharmaceuticals in rivers: Relationships between hazard indexes and aquatic macroinvertebrate diversity indexes in the Llobregat River (NE Spain). Environment International, v. 36, p. 153-162, 2010.

GONÇALVES, D.A.; MARI, J.J.; BOWER, P.; GASK, L.; DOWRICK, C.; TÓFOLI, L.F.; CAMPOS,M.; PORTUGAL, F. B.; BALLESTER, D.; FORTES, S. Brazilian multicentre study of common mental disorders in primary care: rates and related social and demographic factors. Caderno Saúde Pública, v. 30, n. 3, p. 623632, 2014.

GRAVATO, C.; GUIMARÃES, L.; SANTOS, J.; FARIA, M.; ALVES, A.; GUILHERMINO, L. Comparative study about the effects of pollution on glass and yellow eels (Anguilla anguilla) from the estuaries of Minho, Lima and Douro Rivers (NW Portugal). Ecotoxicology and Environmental Safety, v. 73, p. 524-533, 2010.

GUILHERMINO, L.; BARROS, P. ; SILVA, M.C.; SOARES, A.M. V.M. Should the use of inhibition of Cholinesterases as a specific biomarker for organophosphate and carbamate pesticides be questioned? Biomarkers, v. 3, p. 157-151, 1998.

GURKE, R.; ROSSMANN, J.; SCHUBERT, S.; SANDMANN, T.; ROBLER, M.; OERTEL, R.; FAULER, J. Development of a SPE-HPLC - MS / MS method for the determination of most prescribed pharmaceuticals and related metabolites in 
urban sewage samples. Journal of Chromatography B, v. 990, p. 23-30, 2015.

GURKE, R.; ROBLER, M.; MARX, C.; DIAMOND, S.; SCHUBERT, S.; OERTEL, R.; FAULER, J. Occurrence and removal of frequently prescribed pharmaceuticals and corresponding metabolites in wastewater of a sewage treatment plant. Science of the Total Environment, v. 532, p. 762-770, 2015.

HALDER, M.; KIENZLER, A.; WHELAN, M.; WORTH,A. EURL ECVAM Strategy to replace, reduce and refine the use of fish in aquatic toxicity and bioaccumulation testing. JCR Science and Policy Reports, 2014.

HAMMERS-WIRTZ, M.; RATTE, H.T. Offspring fitness in Daphnia: Is the Daphniare production test appropriate for extrapolating effects on the population level? Environmental Toxicology and Chemistry, v. 19, n. 7, p. 1856-1866, 2000.

HAVERROTH, G.M.B., WELANG, C., MOCELIN, R.N., POSTAY, D., BERTONCELLO, K.T., FRANSCESCON, F., ROSEMBERG, D.B., MAGRO. J.D., DALLACORTE, C.L. Copper acutely impairs behavioral function and muscle acetylcholinesteraseactivityinzebrafish (Danio rerio). Ecotoxicology and Environmental Safety, v. 122, p. 440-447, 2015.

HEBERER, T. REDDERSEN, K.; MECHLINSKI, A. From municipal sewage to drinking water: fate and removal of pharmaceutical residues in the aquatic environment in urban areas. Water Science and Technology, v. 46, n. 3, p. 8188, 2002.

HOWE, K.; CLARK, M.D.; TORROJA, C.F.; TORRANCE, J.; BERTHELOT, C.; MUFFATO, M.; COLLINS, J. E.; HUMPHRAY, S.; MCLAREN, K.; MATTHEWS, L.; MCLAREN, S.; SEALY, I.; CACCAMO, M.; CHURCHER, C.; SCOTT, C.; BARRETT, J.C.; KOCH, R.; RAUCH, G.J.; WHITE, S.; CHOW, W.; KILIAN, B.; QUINTAIS, L.T.; GUERRA-ASSUNÇÃO, J.A.; ZHOU, Y.; GU, Y.; YEN, J.; VOGEL, J.-H.; EYRE, T.; REDMOND, S.; BANERJEE, R.; CHI, J.; FU, B.; LANGLEY, E.; MAGUIRE, S.F.; LAIRD, G.K.; LLOYD, D.; KENYON, E.; DONALDSON, S.; SEHRA, H.; ALMEIDA-KING, J.; LOVELAND, J.; TREVANION, S.; JONES, M.; QUAIL, M.; WILLEY, D.; HUNT, A.; BURTON, J.; SIMS, S.; MCLAY, K.; PLUMB, B.; DAVIS, J.; CLEE, C.; OLIVER, K.; CLARK, R.; RIDDLE, C.; ELIOTT, D.; THREADGOLD, G.; HARDEN, G.; WARE, D.; MORTIMER, B.; KERRY, G.; HEATH, P.; PHILLIMORE, B.; TRACEY, A.; CORBY, N.; DUNN, M.; JOHNSON, C.; WOOD, J.; CLARK, S.; PELAN, S.; GRIFFITHS, G.; SMITH, M.; GLITHERO, R.; HOWDEN, P.; BARKER, N.; STEVENS, C.; HARLEY, J.; HOLT, K.; PANAGIOTIDIS, G.; LOVELL, J.; BEASLEY, H.; HENDERSON, C.; GORDON, D.; AUGER, K.; WRIGHT, D.; COLLINS, J.; RAISEN, C.; DYER, L.; LEUNG, K.; ROBERTSON, L.; AMBRIDGE, K.; LEONGAMORNLERT, D.; MCGUIRE, S.; GILDERTHORP, R.; GRIFFITHS, C.; MANTHRAVADI, D.; NICHOL, S.; BARKER, G.; WHITEHEAD, S.; KAY, M.; BROWN, J.; MURNANE, C.; GRAY, E.; HUMPHRIES, M.; SYCAMORE, N.; BARKER, D.; SAUNDERS, D.; WALLIS, J.; BABBAGE, A.; HAMMOND, S.; MASHREGHI-MOHAMMADI, M.; BARR, L.; MARTIN, S.; WRAY, P.; ELLINGTON, A.; MATTHEWS, N.; ELLWOOD, M.; WOODMANSEY, R.; CLARK, G.; COOPER, J.; TROMANS, A.; GRAFHAM, D.; SKUCE, C.; PANDIAN, R.; ANDREWS, R.; HARRISON, E.; KIMBERLEY, A.; GARNETT, J.; FOSKER, N.; 
HALL, R.; GARNER, P.; KELLY, D.; BIRD, C.; PALMER, S.; GEHRING, I.; BERGER, A.; DOOLEY, C.M.; ERSAN-ÜRÜN, Z.; ESER, C.; GEIGER, H.; GEISLER, M.; KAROTKI, L.; KIRN, A.; KONANTZ, J.; KONANTZ, M.; OBERLÄNDER, M.; RUDOLPH-GEIGER, S.; TEUCKE, M.; OSOEGAWA, K.; ZHU, B.; RAPP, A.; WIDAA, S.; LANGFORD, C.; YANG, F.; CARTER, N.P.; HARROW, J.; NING, Z.; HERRERO, J.; SEARLE, S.M.J.; ENRIGHT, A.; GEISLER, R.; PLASTERK, R.H.A.; LEE, C.; WESTERFIELD, M.; DE JONG, P.J.; ZON, L.I.; POSTLETHWAIT, J.H.; NÜSSLEIN-VOLHARD, C.; HUBBARD, T.J.P.; CROLLIUS, H.R.; ROGERS, J.; STEMPLE, D.L. The zebrafish reference genome sequence and its relationship to the human genome. Nature, v. 496, n. 7446, p. 498-503, 17 abr. 2013.

HUNTINGFORD, F.A., ADAMS, C., BRAITHWAITE, V.A., KADRI, S., POTTINGER, T.G., SANDØE, P., TURNBULL, J.F. Current issues in fish welfare. Journal of Fish Biology, 68: 332-372, 2006.

ISBISTER, G.K. Electrocardiogram changes and arrhythmias in venlafaxine overdose. British Journal of Clinical Pharmacology, v. 67, n. 5, p. 572-576, 2009.

ISO. Water Quality - Determination of the Acute Lethal Toxicity of Substances to a Freshwater Fish [Brachydanio rerio Hamilton Buchanan (Teleostei, Cyprinidae)], ISO 7346, 1996.

ISO. Water Quality - Determination of the ocute toxicity of waste water to zebrafish eggs (Danio rerio). ISO 15088, 2007.

JEAN, J.; PERRODIN, Y.; PIVOT, C.; TREPO, D.; PERRAUD, M.; DROGUET, J.; TISSOT-GUERRAZ, F.; LOCHER, F. Identification and prioritization of bioaccumulable pharmaceutical substances discharged in hospital effluents. Journal of Environmental Management, v. 103, p. 113-121, 2012.

JONES, O.A.;LESTER, J.N.; VOULVOULIS, N. Pharmaceuticals: a threat to drinking water? Trends in Biotechnology, v. 23, n. 4, p.163-167, 2005.

KAIS, B.; STENGEL, D.; BATEL, A.; BRAUNBECK, T. Acetylcholinesterase in zebrafish embryos as a tool to identify neurotoxic effects in sediments. Environmental Science and Pollution Research, v. 22, n. 21, p. 16329-16339, 2015.

KASE, R.; KORKARIC, M.; WERNER, I.; AGERSTRANG, M. Criteria for Reporting and Evaluating ecotoxicity Data (CRED): comparison and perception of the Klimisch and CRED methods for evaluating reliability and relevance of ecotoxicity studies. Environmental Sciences Europe, v. 28, p. 7, 2014.

KIMMEL, C.B. et al. Stages of embryonic development of the zebrafish. Developmental Dynamics, v.203, p.253-310, 1995.

KINNEY, C.A.; FURLONG, E.T.; WERNER, S.L.; CAHILL, J.D. presence and distribution of wastewater-derived pharmaceuticals In soil irrigated with reclaimed water. Environmental Toxicology and Chemistry, v. 25, n. 2, p. 317-326, 2006. 
KLEYWEGT, S.; PILEGGI, V.; YANG, P.; HAO, C.; ZHAO, X.; ROCKS, C.; THACH, S.; CHEUNG, P.; WHITEHEAD, B. Pharmaceuticals, hormones and bisphenol $A$ in untreated source and finished drinking water in Ontario, Canada Occurrence and treatment efficiency. Science of the Total Environment,n. 409, p. 1481-1488, 2011.

KOLPIN, D.W.; FURLON, E.; MEYER, M.T.; THURMAN, M.E.; ZAGG, S.D.; BARBER,L.B.; BUXTON, H.T. Pharmaceuticals, Hormones, and Other Organic Wastewater Contaminants in U.S. Streams, 1999-2000: A National Reconnaissance. Environmental Science \& Technology, 2002, 36, 1202-1211, 2002.

KÜSTER, E. Cholin- and carboxylesterase activities in developing zebrafish embryos (Danio rerio) and their potential use for insecticide hazard assessment. Aquatic Toxicology, v. 75, p. 76-85, 2005.

KWON, J.W.; ARMBRUST, K.L. Laboratory persistence and fate of fluoxetine in aquatic environments. Environmental toxicology and chemistry, v. 25, n. 10, p. 2561-2568, 2006.

LAMMER, E., CARR, G.J., WENDLER, K., RAWLINGS, J.M., BELANGER, S.E. BRAUNBECK, T.H. Is the fish embryo toxicity test (FET) with the zebrafish (Danio rerio) a potential alternative for the fish acute toxicity test? Comparative Biochemistry and Physiology, Part C 149: 196-209, 2009a.

LAMMER, E., KAMP, H.G., HISGEN, V., KOCH, M., REINHARD, D., SALINAS, E.R., WENDLER, K., ZOK, S., BRAUNBECK, TH. Development of a flowthrough system for the fish embryo toxicity test (FET) with the zebrafish (Danio rerio). Toxicology in Vitro, 23: 1436-1442, 2009b.

LAUDER, J.M.; SCHAMBRA, U.B. Morphogenetic Roles of Acetylcholine. Environmental Health Perspectives, v. 107, p. 65-69, 1999.

LIN, A.Y.C.; YU, T.H.; LIN, C.F. Pharmaceutical contamination in residential, industrial, and agricultural waste streams: Risk to aqueous environments in Taiwan. Chemosphere, v. 74, p. 131-141, 2008.

LOGARINHO, F.; ROSADO, T.; LOURENC, C. Determination of antipsychotic drugs in hospital and wastewater treatment plant samples by gas chromatography/tandem mass spectrometry. Journal of Chromatography $\mathbf{B}, \mathrm{v}$. 1038, p. 127-135, 2016.

LOOS, R.; CARVALHO, R.; ANTÔNIO, D.C.; COMERO, S.; LOCORO, G.; TAVAZZI, S.; PARACCHINI, B.; GHIANI, M.; LETTIERI, T.; BLAHA, L.; JAROSOVA, B.; VOORSPOELS, S.; SERVAES, K.; HAGLUND, P.; FICK, J.; LINDBERG, R.H.; SCHWESIG, D.; GAWLIK,B.M. EU-wide monitoring survey on emerging polar organic contaminants in wastewater treatment plant effluents. Water Research, v. 47, p. 6475-6487, 2013. 
LUO, Y.; GUO, W.; NGO, H.H.; NGHIEM, L.D.; HAJ, F.I.; ZHANG,J.; LIANG,S.; WANG, X.C. A review on the occurrence of micropollutants in the aquatic environment and their fate and removal during wastewater treatment. Science of the Total Environment, v. 473-474, p. 619-641, 2014.

MARINO, C.L.; JENSEN, B.H. Zebrafish, Danio rerio, Ovaries Spontaneously Contract Just Prior to Lights On. Journal of Behavioral and Neuroscience Research, v. 6, p. 10-14, 2008.

MATZ, C.J.; TREBLE, R.G.; KRONE, P.H. Accumulation and elimination of cadmium in larval stage zebrafish following acute exposure. Ecotoxicology and Environmental Safety, v. 66, p. 44-48, 2007.

MEIRELES, G. Avaliação eco/genotoxicológica dos corantes têxteis Reactive Blue 4 e Reactive Blue 15. 2013.96 f. Dissertação (Mestrado em Ciências) - Faculdade de Ciências Farmacêuticas de Ribeirão Preto - Universidade de São Paulo, Ribeirão Preto, 2013.

MENDONÇA, R.T. A medicalização de conflitos: consumo de ansiolíticos e antidepressivos em grupos populares. 2009. 302f. Tese (Doutorado em Saúde Pública) - Faculdade de Saúde Pública da Universidade de São Paulo, São Paulo, 2009.

METCALFE, C.D.; CHU, S.; JUDT, C.; LI, H.; OAKES,K.D.; SERVOS,M.R.; ANDREWS, D.M. Antidepressants and their metabolites in municipal wastewater, and downstream exposure in an urban watershed. Environmental Toxicology and Chemistry, v. 29, n. 1, p. 79-89, 2010.

MIAO, X.; METCALFE, C.D. Determination of carbamazepine and its metabolites in aqueous samples using liquid chromatography-electrospray tandem mass spectrometry. Analytical Chemistry, v. 75, p. 3731-3738, 2003.

MOERMOND, C.T.A.; KASE, R.; KORKARIC, M.; AGERSTRAND, M. CRED: Criteria for reporting and evaluating ecotoxicity data. Environmental Toxicology and Chemistry, v. 35, p. 1297-1309, 2016.

MOURA, D.S. Avaliação ecotoxicológica de fármacos psicotrópicos e suas possíveis interações com nanomateriais usando embriões de peixe-zebra. 2016. 545 f. Dissertação (Mestrado em Biologia Animal) - Universidade de Brasília, Brasília, 2016.

NAGARNAIK, P.; BATT, A.; BOULANGER, B. Source characterization of nervous system active pharmaceutical ingredients in healthcare facility wastewaters. Journal of Environmental Management, v. 92, p. 872-877, 2011.

NAGEL, R. DarT: The embryo test with the Zebrafish Danio rerio--a general model in ecotoxicology and toxicology. ALTEX: Alternativen zu Tierexperimenten, v. 19, p. 38-48, 2002.

NARBAITZ, R.M.; RANA,D.; DANG, H.T.; MORRISSETTE, J.; MATSUURA, T.; JASIM, S.Y.; TABE, S.; YANG, P.; Pharmaceutical and personal care products 
removal from drinking water by modified cellulose acetate membrane: Field testing. Chemical Engineering Journal, v. 225, p. 848-856, 2013.

NAKADA, N.; KOMORI, K.; SUZUKI, Y.; HOUWA, I.; TANAKA, H. Occurrence of 70 pharmaceutical and personal care products in Tone River basin in Japan. Water Science \& Technology, v. 56, n. 12, p. 133-140, 2007.

NISHIMURA, Y.; MURAKAMI, S.; ASHIKAWA, Y.; SASAGAWA, S.; UEMOTO, N.; SHIMADA, Y.; TANAKA, T. Zebrafish as a systems toxicology model for developmental neurotoxicity testing. Congenital Anomalies, v. 55, n. 1, p. 1-16, 2015.

NÖDLER, K.; VOUTSA, D.; LICHA, T. Polar organic micropollutants in the coastal environment of different marine systems. Marine Pollution Bulletin, v. 85, p. 50$59,2014$.

O'DONNELL, J.M.; SHELTON, R.C. Tratamento farmacológico da depressão e dos transtornos de ansiedade. In: BRUNTON, L.L.; CHABNER, B.A.; KOLLMAN, B.C. As Bases Farmacológicas da Terapêutica de Goodman \& Gilman. $12^{a}$. ed. Porto Alegre: AMGH, 2012.

OECD. OECD Guidelines for the Testing of Chemicals. Section 2: effects on biotic systems test no. 236: fish embryo acute toxicity (FET) test. Organization for Economic Cooperation and Development, Paris, 2013.

OLIVEIRA, T.S.; MURPHY, M.; MENDOLA, N.; WONG, V.; CARLSON, D., WARING., L. Characterization of Pharmaceuticals and Personal Care products in hospital effluent and waste water influent/effluent by direct-injection LC-MSMS. The Science of the Total Environment, v. 518-519, p. 459-478, 2015.

OTTE, J.C.; SCHMIDT, A.D.; HOLLERT, H.; BRAUNBECK, T. Spatio-temporal development of CYP1 activity in early life-stages of zebrafish (Danio rerio). Aquatic Toxicology, v. 100, n. 1, p. 38-50, 2010.

PALMER, P.M.; WILSON, L.R.; O'KEEFE, P.; SHERIDAN, R.; KING, T.; CHEN, C. Sources of pharmaceutical pollution in the New York City Watershed. Science of the Total Environment, v. 394, p. 90-102, 2008.

PAMANJI, R.; BETHU, M.S.; YASHWANTH, B. Developmental toxic effects of monocrotophos, an organophosphorous pesticide , on zebrafish ( Danio rerio ) embryos. Environmental Science and Pollution Research, v. 22, p. 77447753, 2015.

PANULA, P.; CHEN, Y.-C.; PRIYADARSHINI, M.; KUDO, H.; SEMENOVA, S.; SUNDVIK, M.; SALLINEN, $\mathrm{V}$. The comparative neuroanatomy and neurochemistry of zebrafish CNS systems of relevance to human neuropsychiatric diseases. Neurobiology of disease, v. 40, p. 46-57, 2010.

PAPAGEORGIOU, M.; KOSMA, C.; LAMBROPOULOU, D. Seasonal occurrence, removal, mass loading and environmental risk assessment of 55 
pharmaceuticals and personal care products in a municipal wastewater treatment plant in Central Greece. Science of the Total Environment, v. 543, p. 547-569, 2016.

QUINTANA, M.I.; ANDREOLI, S.B.; PELUFFO, M.P.; RIBEIRO, W.S.; FEIJO, M.M.; BRESSAN, R.A.; COUTINHO, E.S.F.; MARI, J.J. Psychotropic drug use in São Paulo, Brazil- An epidemiological survey. Journal PLOS ONE, v. 10, n. 8, p. 1-14, 2015.

REDSHAW, C.H.; COOKE,M.P.; TALBOT, H.M.; MCGRATH, S.; ROWLAND, S.J. Low biodegradability of fluoxetine $\mathrm{HCl}$, diazepam and their human metabolites in sewage sludge-amended soil. Journal of Soils and Sediments, v. 8, n. 4, p. 217-230, 2008.

RICO, E.P.; ROSEMBERG, D.B.; SENGER, M.R.; DE BEM ARIZI, M.; BERNARDI, G.F.; DIAS, R.D.; BOGO, M.R.; BONAN, C.D. Methanol alters ectonucleotidases and acetylcholinesterase in zebrafish brain. Neurotoxicology and Teratology, v. 28, p. 489-496, 2006.

RODRIGUES M.A.P.; LIMA M.S. Modificações nos padrões de consumo de psicofármacos em localidade do Sul do Brasil. Revista Saúde Pública, v. 40, n. 1, p. 107-114, 2006.

RODRÍGUEZ-FUENTES, G.; RUBIO-ESCALANTE, F.J.; NOREÑA-BARROSO, E.; ESCALANTE-HERRERA, K.S.; SCHLENK, D. Comparative Biochemistry and Physiology, Part C Impacts of oxidative stress on acetylcholinesterase transcription, and activity in embryos of zebra fi sh ( Danio rerio) following Chlorpyrifos exposure. Comparative Biochemistry and Physiology, Part C, v. 172-173, p. 19-25, 2015.

ROOS, V.; GUNNARSSON, L.; FICK, J.; LARSSON, D.G.J.; RÚDEN,C. Prioritising pharmaceuticals for environmental risk assessment: Towards adequate and feasible first-tier selection. Science of the Total Environment, v. 421-422, p. 102-110, 2012.

RÚA-GÓMEZ, P.C.; PÜTTMANN, W. Impact of wastewater treatment plant discharge of lidocaine, tramadol, venlafaxine and their metabolites on the quality of surface waters and groundwater. Journal of Environmental Monitoring, v. 14, p. 1391-1399, 2012.

RUSSEL, W.M.S.; BURCH, R.L. 1959. The Principles of Humane Experimental Techniques. Londres: Methuen, 1959.

SALGADO,R.; MARQUES, R.; NORONHA, J.P.; MEXIA, J.T.; OEHMEN, A.; REIS, M.A.M. Assessing the diurnal variability of pharmaceutical and personal care products in a full-scale activated sludge plant. Environmental Pollution, v. 159, p. 2359-2367, 2011.

SANTOS, L.H.M.L.M.; GROS, M.; MOZAZ, S.R.; MATOS, C.D.; PENA, A.; BARCELO, D.; MONTENEGRO, M.C.B.S.M. Contribution of hospital effluents to 
the load of pharmaceuticals in urban wastewaters: Identification of ecologically relevant pharmaceuticals. Science of the Total Environment, v. 461-462, p. 302-316, 2013.

SAUSSEREAU, E.; LACROIX, C.; GUERBET, M.; CELLIER, D.; SPIROUX, J.; GOULLE, J.P. Determination of levels of current drugs in hospital and urban wastewater. Bulletin of environmental contamination and toxicology, v. 91, n. 2, p. 171-6, 2013.

SAWADA, Y.; NASU, H.; MATSUO, H.; OHTANI, H. Relationship between structure and drug-induced parkinsonism. Ann. Pharmacother, v. 34, p. 668, 2000.

SCHULTZ, M.M.; FURLONG, E.T. Trace analysis of antidepressant pharmaceuticals and their select degradates in aquatic matrixes by LC/ESI/MS/MS. Analytical Chemistry, v. 80, n. 5, p. 1756-1762, 2008.

SCHMIDT, S.; BUSCH, W.; ALTENBURGER, R.; KÜSTER, E. Mixture toxicity of water contaminants-effect analysis using the zebrafish embryo assay (Danio rerio). Chemosphere, v. 152, p. 503-512, 2016.

SCOTT, G.R.; SLOMSN, K.A. The effects of environmental pollutants on complex fish behaviour: integrating behavioural and physiological indicators of toxicity. Aquatic Toxicology, v. 68, p. 369-392, 2004.

SELDERSLAGHS, I.W.T.; HOOYBERGHS J.; COEN, W.; WITTERS, E. Locomotor activity in zebrafish embryos: A new method to assess developmental neurotoxicity. Neurotoxicology and Teratology, v. 32, n. 4, p. 460-471, 2010.

SILVA, B.F.; JELIC, A.; LÓPEZ-SERNA, R.; MOZETO, A.A.; PETROVIC, M.; BARCELÓ, D. Occurrence and distribution of pharmaceuticals in surface water, suspended solids and sediments of the Ebro river basin, Spain. Chemosphere, v. 85, p. 1331-1339, 2011.

SILVA, B.; COSTA, F.; NEVES, I.; TAVARES, T. Psychiatric Pharmaceuticals as Emerging Contaminants in Wastewater. Cham: Springer International Publishing, 2015.

SILVA, C.; OLIVEIRA, C.; GRAVATO, C.; ALMEIDA, J.R. Behaviour and biomarkers as tools to assess the acute toxicity of benzo(a)pyrene in the common prawn Palaemon serratus. Marine Environ Res., v. 90, p. 39-46, 2013.

SILVEIRA, M.A.K.; CALDAS, S.S.; GUILHERME, J.R.; COSTA, F.P.; GUIMARÃES, B.S.; CERQUEIRA, M.B.R.; SORAES, B.M.; PRIMEL, E.G. Quantification of pharmaceuticals and personal care product residues in surface and drinking water samples by SPE and LC-ESI-MS/MS. Journal of the Brazilian Chemical Society, v. 24, n. 9, p. 1385-1395, 2013.

SIMAZAKI, D.; HIRAMATSU, S.; FUJIWARA, J.; AKIBA, M.; KUNIKANE, S. Monitoring Priority of Residual Pharmaceuticals in Water Sources and Drinking 
Water in Japan. Journal of Water and Environment Technology, v. 12, n. 3, p. 275-283, 2014.

SPENCE, R.; GERLACH, G.; LAWRENCE, C.; SMITH, C. The behaviour and ecology of the zebrafish, Danio rerio. Biological Reviews, v. 83, n. 1, p. 13-34, 2008.

STACKELBERG, P.E.; FURLONG, E.T.; MEYER, M.T.; ZAUGG, S.D.; HENDERSON, A.K.; REISSMAN, D.B. Persistence of pharmaceutical compounds and other organic wastewater contaminants in a conventional drinking-water treatment plant. Science of the Total Environment, v. 329, p. 99-113, 2004.

STEWART, A.M.; BRAUBACH, O.; SPITSBERGEN, J.; GERLAI, R.; KALUEFF, A.V. Zebrafish models for translational neuroscience research: from tank to bedside. Trends in Neurosciences, v. 37, n. 5, p. 264-278, 2014.

STYRISHAVE, B.; HALLING-SORENSEN, B; INGERSLEV, F. Environmental risk assessment of three selective serotonin reuptake inhibitors in the aquatic environment: a case study including a cocktail scenario. Environmental Toxicology and Chemistry, v. 30, n. 1, p. 254-261, 2011.

SUI, Q.; HUANG, J.; DENG, S.; YU, G.; FAN, Q. Occurrence and removal of pharmaceuticals, caffeine and DEET in wastewater treatment plants of Beijing, China. Journal Water Research, v. 44, p. 417-426, 2010.

TEIJON, G.; CANDELA, L.; TAMOH, K.; MOLINA-DIAZ, A.; FERNÁNDEZ-ALBA, A.R. Occurrence of emerging contaminants, priority substances (2008/105/CE) and heavy metals in treated wastewater and groundwater at Depurbaix facility (Barcelona, Spain). Science of the Total Environment, v. 408, p. 3584-3595, 2010.

TAYLOR, P. Agentes anticolinesterásicos. In: BRUNTON, L.L.; CHABNER, B.A.; KOLLMAN, B.C. As Bases Farmacológicas da Terapêutica de Goodman \& Gilman. $12^{\text {a }}$. ed. Porto Alegre: AMGH, 2012.

TEIXIDÓ, E.; PIQUÉ, E.; GÓMES-CATALÁN, J.; LOBET, J.M. Assessment of developmental delay in the zebrafish embryo teratogenicity assay. Toxicology in Vitro, v. 27, p. 469-478, 2012.

THUMMEL, K.E.; SHEN, D.D.; ISOHERRANEN, N. Planejamento e otimização de esquemas posológicos: dados farmacocinéticos. In: BRUNTON, L.L.; CHABNER, B.A.; KOLLMAN, B.C. As Bases Farmacológicas da Terapêutica de Goodman \& Gilman. 12 ${ }^{\text {a }}$. ed. Porto Alegre: AMGH, 2012.

TRUONG, L.; HARPER, S.L.; TANGUAY, R.L. Evaluation of embryotoxicity using the zebrafish model. Methods in molecular biology, v. 691, p. 1-8, 2011. 
UMBUZEIRO, G.A.; KUMMROW,F.; REIS, F.F.C. Toxicologia, padrões de qualidade de água e a legislação. Revista de Gestão Integrada em Saúde do Trabalho e Meio Ambiente, v. 5, n. 1, 2010.

UN. United Nations News Centre. UN health agency reports depression now 'leading cause of disability worldwide, 23 february 2017. Disponível em:< http://www.un.org/apps/news/story.asp?Newsld=56230\#.WmFV4a6nHIX>.

Acesso em: 11 de dezembro de 2017.

UNIÃO EUROPEIA. Directiva 2010/63/UE do Parlamento Europeu e do Conselho. Jornal Oficial da União Europeia, 20 out. 2010.

US Environmental Protection Agency. Evaluation guidelines for ecological toxicity data in the open literature. Procedures for screening, reviewing, and using published open literature toxicity data in ecological risk assessments, 2011. Disponível em <http://www.epa.gov/pesticide-science-and-assessing-pesticiderisks/evaluation-guidelines-ecological-toxicity-data-open>. Acesso em: 20 de março de 2017.

VILLANUEVA, C.M.; KOGEVINAS, M.; CORDIER, S.; TEMPLETON, M.R.; VERMEULEN, R.; NUCKOLS, J.R.; NIEWENHUIJSEN, M.J.; LEVALLOIS, P.; Assessing exposure and health consequences of chemicals in drinking water: current state of knowledge and research needs. Environmental Health Perspectives, v. 122, n. 3, p. 213-221, 2014.

WHO. World Health Organization. Depression: Let's talk. 1-year on. World Health Day, 7 April 2017. Disponível em: <http://www.who.int/campaigns/worldhealth-day/2017/en/>. Acesso em: 11 de dezembro de 2017.

WHO. World Health Organization. Mental Health Atlas-2011 country profiles, Brazil. Disponível em: < http://www.who.int/mental_health/ evidence/atlas/profiles/en/ >. Acessado em: 11 de dezembro de 2017.

WU, C.; WITTER, J.D.; SPONGBERG, A.L.; CZAJKOWSKI, K.P. Occurrence of selected pharmaceuticals in an agricultural landscape, western Lake Erie basin. Water Research, v. 43, p. 3407-3416, 2009.

YAMASHITA, A.; INADA, H.; CHIHARA, K.; YAMADA, T.; DEGUCHI, J.; FUNABASHI, $\mathrm{H}$. Improvement of the evaluation method for teratogenicity using zebrafish embryos. Journal of Toxicological Sciences, v. 39, n. 3, p. 453-464, 2014.

YUAN, S.; JIANG, X.; ZHANG, H., ZHENG, S. Detection, occurrence and fate of 22 psychiatric pharmaceuticals in psychiatric hospital and municipal wastewater treatment plants in Beijing, China. Chemosphere, v. 90, n. 10, p. 2520-2525, 2013.

ZORITA, S.; MARTENSSON, L.; MATHIASSON, L. Occurrence and removal of pharmaceuticals in a municipal sewage treatment system in the south of Sweden. Science of the Total Environment, v. 407, n. 8, p. 2760-2770, 2009. 